Supporting Information for:

\title{
Block Copolymer Amphiphile Phase Diagrams by High- Throughput Transmission Electron Microscopy
}

\author{
Mollie A. Touve, ${ }^{\mathrm{a}, \mathrm{d}} \S$ Daniel B. Wright, ${ }^{\mathrm{a}, \mathrm{b}, \mathrm{c}, \mathrm{d}} \S$ Chen Mu, ${ }^{\mathrm{e}}$ Hao Sun, ${ }^{\mathrm{a}, \mathrm{b}, \mathrm{c}, \mathrm{d}}$ Chiwoo Park, ${ }^{\mathrm{e}}$ and Nathan C. \\ Gianneschi*a,b,c,d \\ ${ }^{a}$ Department of Chemistry, ${ }^{b}$ Department of Materials Science and Engineering, 'Department of Biomedical \\ Engineering, ${ }^{\mathrm{d}}$ International Institute for Nanotechnology, Chemistry of Life Processes Institute, and Simpson Querrey \\ Institute, Northwestern University, 2145 Sheridan Road, Evanston, Illinois 60208-3113, United States of America \\ eDepartment of Industrial \& Manufacturing Engineering, Florida State University, Tallahassee, Florida 32310, United \\ States
}




\section{Contents}

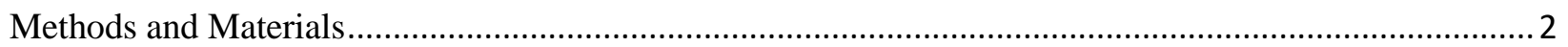

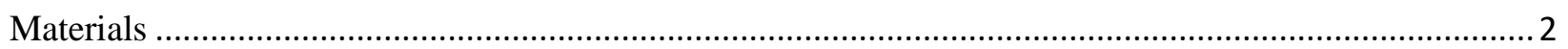

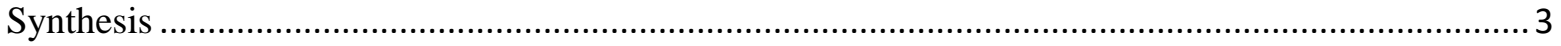

Synthesis of poly(DMAEMA)-macroCTA ……........................................................................... 3

General procedure for UV-initiated iniferter polymerization ....................................................... 3

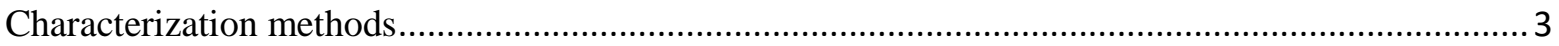

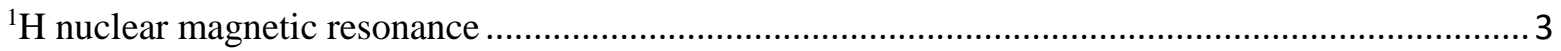

Size exclusion chromatography multi-angle light scattering. ................................................... 3

Standard single-sample dry-state transmission electron microscopy (TEM) ........................................... 4

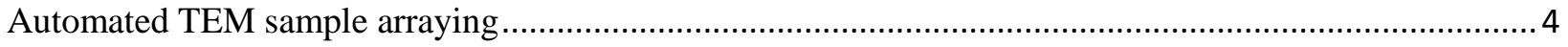

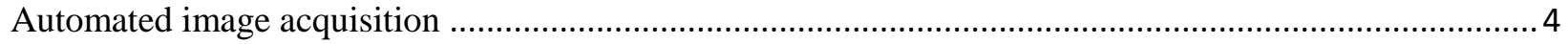

Figure S1. Automated droplet dispensing system (sciTEM) ............................................................ 5

Figure S2. Fiducial-marked TEM grid before and after droplet dispensing .......................................... 6

Method for Shape Identification and Classification............................................................................ 7

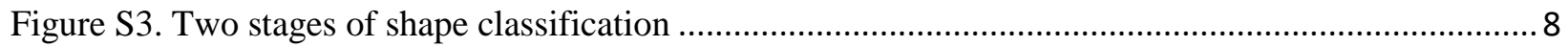

Table S1. Size-exclusion chromatography (SEC) characterization of PEG- $b$-PHPMA block copolymers.. 9

Table S2. SEC-MALS and ${ }^{1} \mathrm{H}$ NMR characterization of PDMAEMA-macroCTA ................................. 10

Table S3. Size-exclusion chromatography (SEC) characterization of PDMAEMA- $b$-PHPMA block

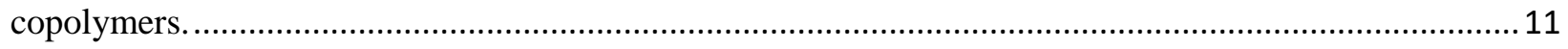

Table S4. Automated shape classification for PEG- $b$-PHPMA nanostructures...................................... 12

Table S5. Automated shape classification for PDMAEMA- $b$-PHPMA nanostructures............................13

Figure S4. Companion to Figure 7 of the main text............................................................................ 14

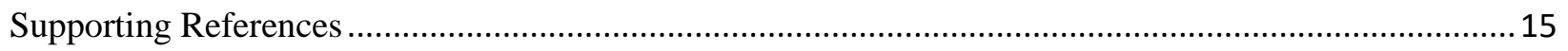

\section{Methods and Materials}

\section{Materials}

PEG macro-Chain-Transfer Agent (PEG-macroCTA) was synthesised according to previous literature reports. ${ }^{1}$ All other materials were used as received from Aldrich, Fluka, Apeiron catalyst or Acros. 


\section{Synthesis}

\section{Synthesis of poly(DMAEMA)-macroCTA}

A solution of 2-(dimethylamino)ethyl methacrylate (DMAEMA), 25 equivalents, 0.1 equivalents of AIBN and 1 equivalent of 4-cyano-4-[(dodecylsulfanylthiocarbonyl)sulfanyl] pentanoic acid was added to a dry ampoule containing a stir bar. The solution was degassed by purging with nitrogen for 20 minutes, sealed and placed in a pre-heated oil bath at $70{ }^{\circ} \mathrm{C}$. After 8 hours the conversion reached more than $99 \%$. The polymerization was quenched by liquid nitrogen and opened to air. The crude reaction was diluted with the minimum amount of dichloromethane $\left(\mathrm{CH}_{2} \mathrm{Cl}_{2}\right)$, and the solution was precipitated into cold hexane. The polymer was isolated by filtration. Precipitation was repeated two more times to afford a yellow macroCTA polymer powder.

\section{General procedure for UV-initiated iniferter polymerization}

In a 96-well plate, macroCTA (either PDMAEMA-macroCTA or PEG-macroCTA) (1 equiv.) degassed hydroxypropyl methacrylate (equiv. dependent on targeted block length) and degassed $18 \mathrm{~m} \Omega$ water (volume dependent on solids wt \%) were added, reactions performed at $100 \mu \mathrm{L}$, under a nitrogen atmosphere. The 96-well plate was sealed and illuminated for $60 \mathrm{~min}$ at $\lambda 365 \mathrm{~nm}$. After $60 \mathrm{~min}$, illumination was stopped and the well plate was opened to air to quench the polymerization.

\section{Characterization methods}

\section{${ }^{1} \mathrm{H}$ nuclear magnetic resonance}

${ }^{1} \mathrm{H}$ Nuclear magnetic resonance (NMR) spectra were recorded on a Bruker Advance III Au 400 (400 MHz)

in N,N-Dimethyl-d6-formamide. Chemical shifts are given in ppm downfield from tetramethylsilane (TMS).

\section{Size exclusion chromatography multi-angle light scattering}

Size exclusion chromatography multi-angle light scattering (SEC-MALS) measurements were performed on a set of Phenomenex Phenogel 5u, 1K-75K, 300 x $7.80 \mathrm{~mm}$ in series with a Phenomex Phenogel 5u, 10K-1000K, 300 x $7.80 \mathrm{~mm}$ columns with HPLC grade solvents as eluents: dimethylformamide (DMF) with $0.05 \mathrm{M}$ of $\mathrm{LiBr}$ at $40^{\circ} \mathrm{C}$ with a Wyatt Dawn Heleos II and a Optilab T-rEX. The molecular weights of the synthesized polymers were calculated using Astra software after normalizing the detectors with a 30K PS standard. 


\section{Standard single-sample dry-state transmission electron microscopy (TEM)}

Small aliquots $(4 \mu \mathrm{L})$ of sample were applied onto 400 mesh carbon grids (Ted Pella, INC.) that had been glow discharged using a PELCO easiGlow glow discharge unit for $90 \mathrm{~s}$. Excess sample solution was wicked away with filter paper, then the grid was stained with $1 \%(\mathrm{w} / \mathrm{v})$ uranyl acetate, washed with water, and allowed to dry. TEM imaging was conducted on a JEM-ARM300F (JEOL, Ltd., Tokyo, Japan) operated at $300 \mathrm{keV}$. Micrographs were recorded on a 2k x 2k Gatan OneView-IS CCD camera (Gatan Inc., Pleasanton, CA, USA) using Gatan Digital Micrograph image acquisition software (Roper Technologies, Sarasota, FL).

\section{Automated TEM sample arraying}

Samples were prepared as described in a 96 well plate. Samples of higher viscosity (e.g., Compositions \# 35-45) were diluted in water to decrease viscosity and concentration. $20 \mu \mathrm{L}$ of each sample were loaded into a fresh 96 well plate, which was then loaded into a sciTEM instrument (SCIENION AG, Berlin, Germany) under ambient conditions. A Reference 200 mesh (AHERA) carbon film copper grid with alphanumeric coding (Ted Pella, Inc., Redding, CA, USA) was glow discharged for $10 \mathrm{~s}$ at $30 \mathrm{~mA}$ (Pelco EasiGlow), then placed onto a glass slide. Locations on the reference grid were chosen for sample dispensing, then the automated droplet dispensing system deposited a $250 \mathrm{pL}$ droplet of each sample at predefined locations. Each droplet dried immediately after dispensing. Between each dispense, automatically, the nozzle of the system was thoroughly cleaned. After a full dispense, the grid was removed and imaged by TEM.

\section{Automated image acquisition}

Automated TEM sample navigation, image acquisition, and image alignment were performed with the microscope control program SerialEM² on a JEM-ARM300F (JEOL, Ltd., Tokyo, Japan) operated at 300 keV. Micrographs were recorded on a 2k x 2k Gatan OneView-IS CCD camera (Gatan Inc., Pleasanton, CA, USA). 


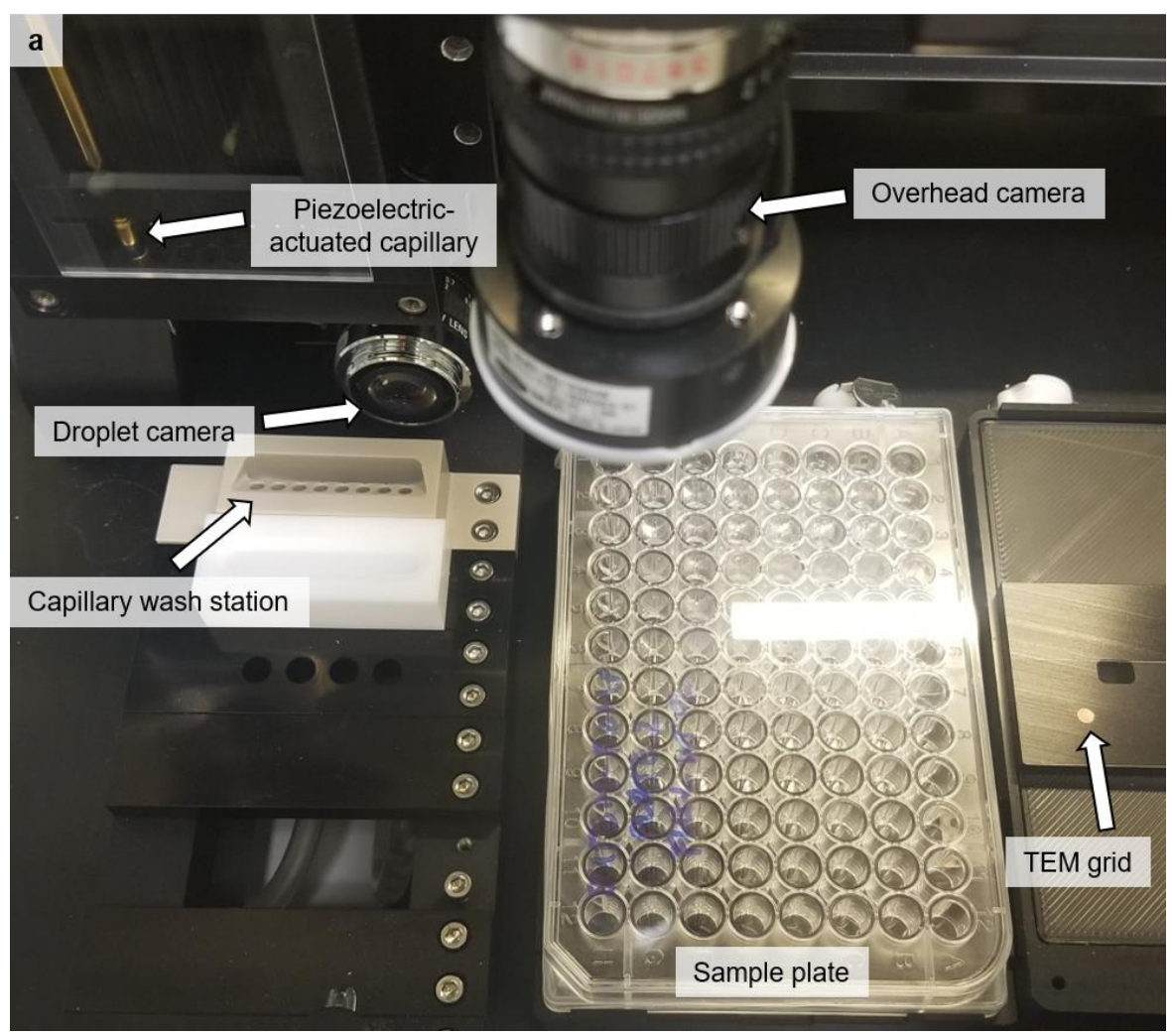

Figure S1. Automated droplet dispensing system (sciTEM). (a) Digital picture of sciTEM setup for arraying samples from a 96-well plate onto a TEM grid, with major components labeled. 


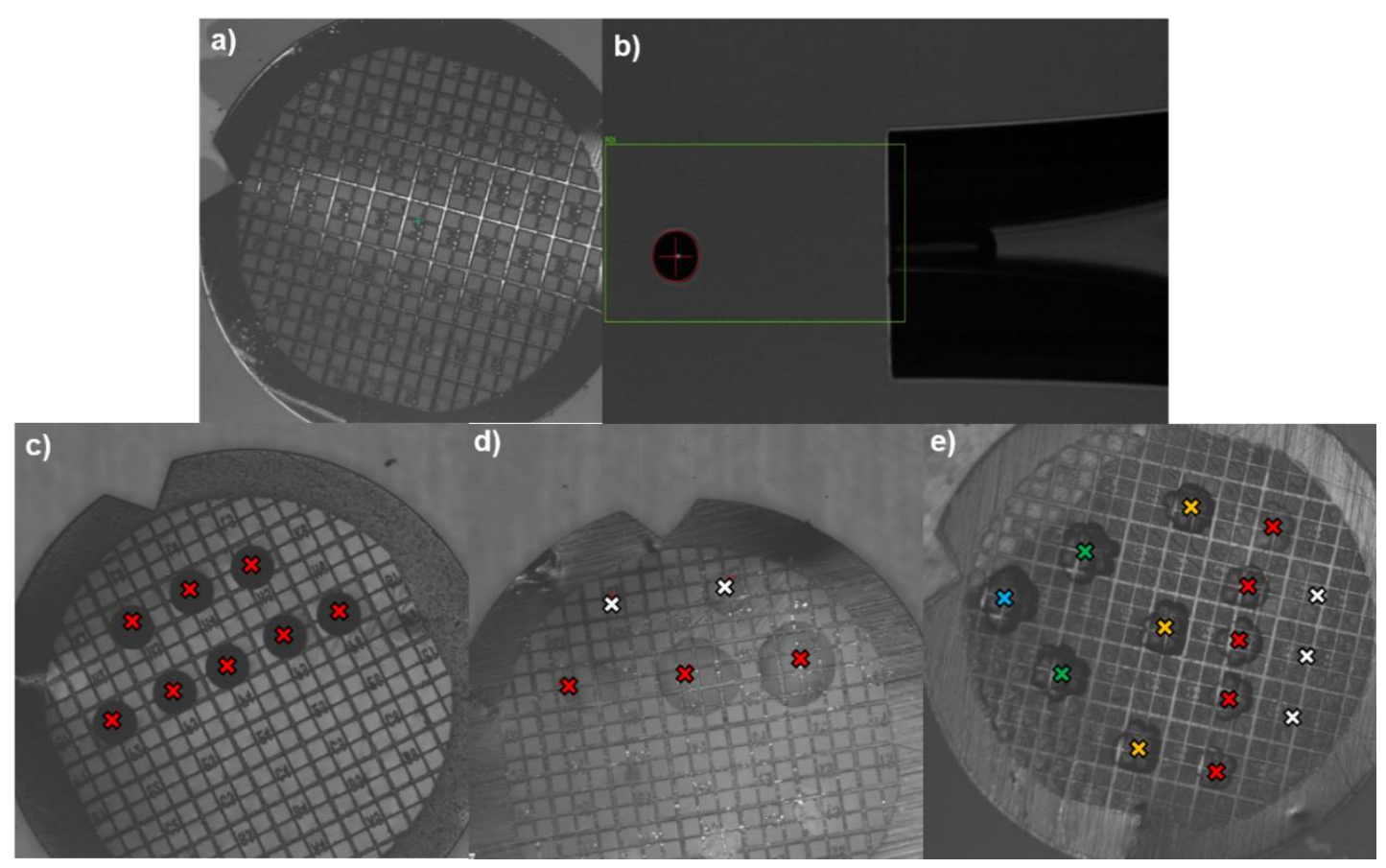

Figure S2. Fiducial-marked TEM grid before and after droplet dispensing. (a) Optical picture of TEM grid before dispensing, taken by a sciTEM camera. (b) Array of $320 \mathrm{pL}$ sample droplets being dispensed by the sciTEM. (c) Optical picture of TEM grid which had not been glow-discharged after dispensing an array of $320 \mathrm{pL}$ water droplets (dispense locations marked in red). (d) Optical picture of TEM grid that was glow-discharged for 2 min prior to dispensing an array of 2 x $320 \mathrm{pL}$ ( $640 \mathrm{pL}$ total volume, location marked in white) or $5 \times 320 \mathrm{pL}$ ( $2 \mathrm{~nL}$ total volume, location marked in red) water droplets. (e) Optical picture of TEM grid that was glow-discharged for $30 \mathrm{~s}$ prior to dispensing of 1 x $320 \mathrm{pL}$ droplet (white), 2 × $320 \mathrm{pL}$ droplets (red), 3 x 320 pL droplets (orange), 4 × 320 pL droplets (green), 5 x 320 pL droplets (blue). Grids shown in (c-e) were placed in a humidity chamber so that droplets did not evaporate immediately upon dispensing. 


\section{Method for Shape Identification and Classification}

A TEM micrograph (Figure S3a) was first analyzed by a robust image binarization method $^{3}$ to segment the

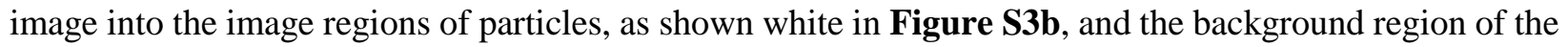
image, as shown black in Figure S3c. The image regions of micelles were classified into three types, spheres, vesicles and worm-likes, by the outlines and interior intensity patterns of the image regions (Figure S3d). We first applied the elastic curve-based shape clustering algorithm ${ }^{4}$ on the outlines to group the outlines into two distinct groups, spherical outlines and non-spherical outlines. The clustering method is an unsupervised method of grouping the outlines by their shapes, so no training data is required. Once the clustering is finished, the operator can assign the groups as either spherical or non-spherical and nonconvex. The regions of non-spherical outlines are labelled as worm-like micelles. The regions of spherical outlines are further subcategorized by the interior intensity patterns of the regions into two subtypes, "spherical outline + uniform intensity" and "spherical outline + nonuniform intensity". Specifically, we extracted the radial intensity profile of each micelle region detected, where the radial intensity profile is a plot of the image intensity versus the distance from the micelle centroid. The degree of the uniformity was defined by the variance of the intensity profile, which is compared with a preset threshold to classify it into uniform and non-uniform. The uniform particles are labelled as spherical micelles, while the non-uniform particles are labelled as vesicles. The percentages of the worm-like micelles, spherical micelles and vesicles in each micrograph were calculated. 

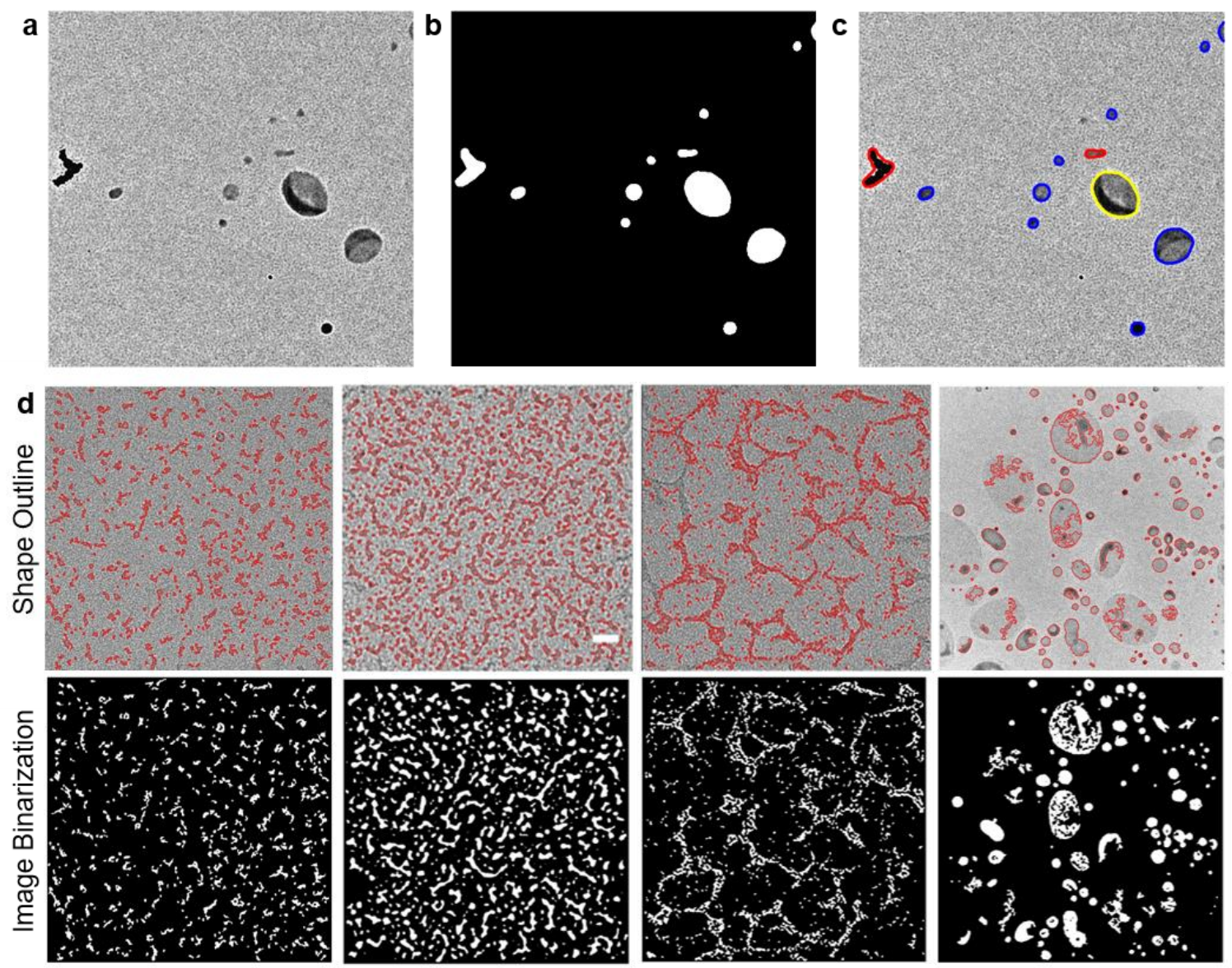

Figure S3. Two stages of shape classification. In the first stage, an input microscope image as shown in (a) is segmented into the regions of particles (shown in white in (b)) and the background region (black in (b)). The second stage classify the regions of particles into three types by the outlines and interior image intensity patterns of the regions as shown in (c), where spheres are in blue, worm-like micelles are in red and vesicles are in yellow. (d) Additional examples of shape outlines (top row) and image binarization (bottom row). 


\begin{tabular}{|c|c|c|c|c|c|c|c|c|c|}
\hline Polymer & $\begin{array}{c}\text { Target } \\
N_{\text {PHPMA }}\end{array}$ & $\begin{array}{c}\text { Solids } \\
\%\end{array}$ & $\begin{array}{l}M_{\mathrm{n}} \mathrm{SEC} \\
(\mathrm{kDa})\end{array}$ & Đ & Polymer & $\begin{array}{c}\text { Target } \\
N_{\text {PHPMA }}\end{array}$ & $\begin{array}{c}\text { Solids } \\
\%\end{array}$ & $\begin{array}{c}M_{\mathrm{n}} \mathrm{SEC} \\
(\mathrm{kDa})\end{array}$ & 甲 \\
\hline 1 & 50 & 10 & 15.0 & 1.22 & 28 & 175 & 20 & 37.8 & 1.22 \\
\hline 2 & 50 & 15 & 15.4 & 1.29 & 29 & 175 & 25 & 36.9 & 1.26 \\
\hline 3 & 50 & 20 & 14.7 & 1.23 & 30 & 175 & 30 & 38.2 & 1.13 \\
\hline 4 & 50 & 25 & 16.2 & 1.25 & 31 & 200 & 10 & 40.0 & 1.27 \\
\hline 5 & 50 & 30 & 14.5 & 1.30 & 32 & 200 & 15 & 41.0 & 1.26 \\
\hline 6 & 75 & 10 & 19.4 & 1.31 & 33 & 200 & 20 & 42.1 & 1.15 \\
\hline 7 & 75 & 15 & 19.7 & 1.28 & 34 & 200 & 25 & 40.1 & 1.31 \\
\hline 8 & 75 & 20 & 20.1 & 1.21 & 35 & 200 & 30 & 41.1 & 1.19 \\
\hline 9 & 75 & 25 & 22.3 & 1.17 & 36 & 225 & 10 & 46.1 & 1.18 \\
\hline 10 & 75 & 30 & 20.2 & 1.28 & 37 & 225 & 15 & 45.2 & 1.34 \\
\hline 11 & 100 & 10 & 23.5 & 1.15 & 38 & 225 & 20 & 46.2 & 1.34 \\
\hline 12 & 100 & 15 & 22.9 & 1.56 & 39 & 225 & 25 & 45.8 & 1.13 \\
\hline 13 & 100 & 25 & 24.0 & 1.31 & 40 & 225 & 30 & 44.3 & 1.25 \\
\hline 14 & 100 & 30 & 25.1 & 1.21 & 41 & 300 & 10 & 58.4 & 1.33 \\
\hline 15 & 100 & 20 & 23.7 & 1.19 & 42 & 300 & 15 & 58.5 & 1.11 \\
\hline 16 & 125 & 10 & 28.2 & 1.12 & 43 & 300 & 20 & 60.3 & 1.28 \\
\hline 17 & 125 & 15 & 28.0 & 1.13 & 44 & 300 & 25 & 65.2 & 1.17 \\
\hline 18 & 125 & 25 & 28.4 & 1.27 & 45 & 300 & 30 & 59.0 & 1.30 \\
\hline 19 & 125 & 20 & 27.6 & 1.19 & & & & & \\
\hline 20 & 125 & 30 & 31.1 & 1.18 & & & & & \\
\hline 21 & 150 & 10 & 32.3 & 1.34 & & & & & \\
\hline 22 & 150 & 25 & 31.4 & 1.23 & & & & & \\
\hline 23 & 150 & 15 & 31.3 & 1.21 & & & & & \\
\hline 24 & 150 & 20 & 35.4 & 1.28 & & & & & \\
\hline 25 & 150 & 30 & 35.5 & 1.29 & & & & & \\
\hline 26 & 175 & 10 & 36.7 & 1.23 & & & & & \\
\hline 27 & 175 & 15 & 36.6 & 1.19 & & & & & \\
\hline
\end{tabular}

Table S1. Size-exclusion chromatography (SEC) characterization of PEG-b-PHPMA block copolymers. 


\begin{tabular}{cccc} 
Polymer & $\begin{array}{c}M_{\mathrm{n} \mathrm{SEC}}{ }^{\mathrm{a}} \\
(\mathrm{kDa})\end{array}$ & $\begin{array}{c}M_{\mathrm{n} \mathrm{NMR}}{ }^{\mathrm{b}} \\
(\mathrm{kDa})\end{array}$ & $Ð^{\mathrm{a}}$ \\
\hline P(DMAEMA) & 5.2 & 4.3 & 1.2 \\
\hline
\end{tabular}

${ }^{\mathrm{a}}$ From SEC based on poly(styrene) standards in DMF. ${ }^{\mathrm{b}}$ Determined by end-group analysis from ${ }^{1} \mathrm{H}$ NMR spectroscopy.

Table S2. SEC-MALS and ${ }^{1} \mathrm{H}$ NMR characterization of PDMAEMA-macroCTA. 


\begin{tabular}{|c|c|c|c|c|c|c|c|c|c|}
\hline Polymer & $\begin{array}{l}\text { Target } \\
N_{\text {PHPMA }}\end{array}$ & $\begin{array}{c}\text { Solids } \\
\%\end{array}$ & $\begin{array}{c}M_{\mathrm{n}} \mathrm{SEC} \\
(\mathrm{kDa})\end{array}$ & Ð & Polymer & $\begin{array}{l}\text { Target } \\
N_{\text {PHPMA }}\end{array}$ & $\begin{array}{c}\text { Solids } \\
\%\end{array}$ & $\begin{array}{c}M_{\mathrm{n}} \\
\mathrm{SEC} \\
(\mathrm{kDa})\end{array}$ & Đ \\
\hline 1 & 50 & 5 & 13.8 & 1.26 & 28 & 175 & 15 & 34.6 & 1.23 \\
\hline 2 & 50 & 10 & 13.2 & 1.20 & 29 & 175 & 20 & 33.9 & 1.31 \\
\hline 3 & 50 & 15 & 12.1 & 1.31 & 30 & 175 & 25 & 37.9 & 1.15 \\
\hline 4 & 50 & 20 & 14.1 & 1.29 & 31 & 175 & 30 & 39.7 & 1.19 \\
\hline 5 & 50 & 25 & 11.3 & 1.18 & 32 & 200 & 10 & 38.8 & 1.27 \\
\hline 6 & 50 & 30 & 18.1 & 1.19 & 33 & 200 & 15 & 41.1 & 1.34 \\
\hline 7 & 75 & 10 & 19.1 & 1.24 & 34 & 200 & 20 & 41.2 & 1.18 \\
\hline 8 & 75 & 15 & 17.2 & 1.14 & 35 & 200 & 25 & 41.1 & 1.28 \\
\hline 9 & 75 & 20 & 18.8 & 1.13 & 36 & 200 & 30 & 44.0 & 1.27 \\
\hline 10 & 75 & 25 & 21.1 & 1.30 & 37 & 225 & 10 & 41.0 & 1.29 \\
\hline 11 & 75 & 30 & 22.4 & 1.14 & 38 & 225 & 15 & 43.2 & 1.14 \\
\hline 12 & 100 & 10 & 22.8 & 1.28 & 39 & 225 & 20 & 44.6 & 1.21 \\
\hline 13 & 100 & 15 & 24.0 & 1.27 & 40 & 225 & 25 & 47.8 & 1.20 \\
\hline 14 & 100 & 20 & 23.5 & 1.25 & 41 & 225 & 30 & 57.0 & 1.11 \\
\hline 15 & 100 & 25 & 24.5 & 1.24 & 42 & 300 & 10 & 59.2 & 1.34 \\
\hline 16 & 100 & 30 & 26.7 & 1.33 & 43 & 300 & 15 & 56.9 & 1.25 \\
\hline 17 & 125 & 10 & 28.3 & 1.17 & 44 & 300 & 20 & 56.5 & 1.11 \\
\hline 18 & 125 & 15 & 28.7 & 1.15 & 45 & 300 & 25 & 65.7 & 1.32 \\
\hline 19 & 125 & 20 & 28.4 & 1.21 & & & & & \\
\hline 20 & 125 & 25 & 27.7 & 1.14 & & & & & \\
\hline 21 & 125 & 30 & 31.1 & 1.23 & & & & & \\
\hline 22 & 150 & 10 & 30.3 & 1.25 & & & & & \\
\hline 23 & 150 & 15 & 30.1 & 1.19 & & & & & \\
\hline 24 & 150 & 20 & 33.3 & 1.18 & & & & & \\
\hline 25 & 150 & 25 & 33.0 & 1.26 & & & & & \\
\hline 26 & 150 & 30 & 35.4 & 1.22 & & & & & \\
\hline 27 & 175 & 10 & 32.5 & 1.26 & & & & & \\
\hline
\end{tabular}

Table S3. Size-exclusion chromatography (SEC) characterization of PDMAEMA- $b$-PHPMA block copolymers. 


\begin{tabular}{|c|c|c|c|c|c|c|c|}
\hline Polymer & $\begin{array}{l}\text { Percent } \\
\text { Spherical } \\
\text { Micelles }\end{array}$ & $\begin{array}{c}\text { Percent } \\
\text { Worm-like } \\
\text { Micelles }\end{array}$ & $\begin{array}{l}\text { Percent } \\
\text { Vesicles }\end{array}$ & Polymer & $\begin{array}{l}\text { Percent } \\
\text { Spherical } \\
\text { Micelles }\end{array}$ & $\begin{array}{c}\text { Percent } \\
\text { Worm-like } \\
\text { Micelles }\end{array}$ & $\begin{array}{l}\text { Percent } \\
\text { Vesicles }\end{array}$ \\
\hline 1 & 0.32 & 0.68 & 0.00 & 24 & 0.26 & 0.74 & 0.00 \\
\hline 2 & 0.30 & 0.70 & 0.00 & 25 & 0.24 & 0.76 & 0.00 \\
\hline 3 & 0.17 & 0.83 & 0.00 & 26 & 0.21 & 0.79 & 0.00 \\
\hline 4 & 0.20 & 0.80 & 0.00 & 27 & 0.28 & 0.72 & 0.00 \\
\hline 5 & 0.24 & 0.76 & 0.00 & 28 & 0.26 & 0.74 & 0.00 \\
\hline 6 & 0.21 & 0.79 & 0.00 & 29 & 0.12 & 0.88 & 0.00 \\
\hline 7 & 0.23 & 0.77 & 0.00 & 30 & 0.26 & 0.74 & 0.00 \\
\hline 8 & 0.17 & 0.83 & 0.00 & 31 & 0.24 & 0.76 & 0.00 \\
\hline 9 & 0.20 & 0.80 & 0.00 & 32 & 0.21 & 0.79 & 0.00 \\
\hline 10 & 0.21 & 0.79 & 0.00 & 33 & 0.19 & 0.81 & 0.00 \\
\hline 11 & 0.23 & 0.77 & 0.00 & 34 & 0.25 & 0.75 & 0.00 \\
\hline 12 & 0.19 & 0.80 & 0.01 & 35 & 0.13 & 0.87 & 0.00 \\
\hline 13 & 0.29 & 0.71 & 0.00 & 36 & 0.19 & 0.81 & 0.00 \\
\hline 14 & 0.22 & 0.78 & 0.00 & 37 & 0.18 & 0.82 & 0.00 \\
\hline 15 & 0.20 & 0.80 & 0.00 & 38 & 0.20 & 0.80 & 0.00 \\
\hline 16 & 0.29 & 0.70 & 0.00 & 39 & 0.22 & 0.78 & 0.00 \\
\hline 17 & 0.22 & 0.78 & 0.00 & 40 & 0.33 & 0.67 & 0.00 \\
\hline 18 & 0.24 & 0.76 & 0.00 & 41 & 0.72 & 0.09 & 0.18 \\
\hline 19 & 0.29 & 0.71 & 0.00 & 42 & 0.89 & 0.03 & 0.08 \\
\hline 20 & 0.16 & 0.84 & 0.00 & 43 & 0.11 & 0.00 & 0.89 \\
\hline 21 & 0.28 & 0.72 & 0.00 & 44 & 0.58 & 0.05 & 0.37 \\
\hline 22 & 0.28 & 0.72 & 0.00 & 45 & 0.73 & 0.02 & 0.25 \\
\hline 23 & 0.25 & 0.75 & 0.00 & & & & \\
\hline
\end{tabular}

Table S4. Automated shape classification for PEG-b-PHPMA nanostructures. 


\begin{tabular}{|c|c|c|c|c|c|c|c|}
\hline Polymer & $\begin{array}{l}\text { Percent } \\
\text { Spherical } \\
\text { Micelles }\end{array}$ & $\begin{array}{c}\text { Percent } \\
\text { Worm-like } \\
\text { Micelles }\end{array}$ & $\begin{array}{l}\text { Percent } \\
\text { Vesicles }\end{array}$ & Polymer & $\begin{array}{l}\text { Percent } \\
\text { Spherical } \\
\text { Micelles }\end{array}$ & $\begin{array}{c}\text { Percent } \\
\text { Worm-like } \\
\text { Micelles }\end{array}$ & $\begin{array}{l}\text { Percent } \\
\text { Vesicles }\end{array}$ \\
\hline 1 & 1.00 & 0.00 & 0.00 & 24 & 0.20 & 0.80 & 0.00 \\
\hline 2 & 0.16 & 0.84 & 0.00 & 25 & 0.10 & 0.90 & 0.00 \\
\hline 3 & 0.29 & 0.71 & 0.00 & 26 & 0.05 & 0.95 & 0.00 \\
\hline 4 & 0.09 & 0.91 & 0.00 & 27 & 0.28 & 0.72 & 0.00 \\
\hline 5 & 0.09 & 0.91 & 0.00 & 28 & 0.05 & 0.95 & 0.00 \\
\hline 6 & 0.19 & 0.81 & 0.00 & 29 & 0.05 & 0.95 & 0.00 \\
\hline 7 & 0.27 & 0.73 & 0.00 & 30 & 0.10 & 0.90 & 0.00 \\
\hline 8 & 0.21 & 0.79 & 0.00 & 31 & 0.12 & 0.88 & 0.00 \\
\hline 9 & 0.18 & 0.82 & 0.00 & 32 & 0.10 & 0.90 & 0.00 \\
\hline 10 & 0.21 & 0.79 & 0.00 & 33 & 0.12 & 0.88 & 0.00 \\
\hline 11 & 0.10 & 0.90 & 0.00 & 34 & 0.08 & 0.92 & 0.00 \\
\hline 12 & 0.30 & 0.70 & 0.00 & 35 & 0.10 & 0.90 & 0.00 \\
\hline 13 & 0.17 & 0.83 & 0.00 & 36 & 0.09 & 0.91 & 0.00 \\
\hline 14 & 0.16 & 0.84 & 0.00 & 37 & 0.08 & 0.92 & 0.00 \\
\hline 15 & 0.27 & 0.73 & 0.00 & 38 & 0.04 & 0.96 & 0.00 \\
\hline 16 & 0.34 & 0.66 & 0.00 & 39 & 0.09 & 0.91 & 0.00 \\
\hline 17 & 0.23 & 0.77 & 0.00 & 40 & 0.04 & 0.96 & 0.00 \\
\hline 18 & 0.20 & 0.80 & 0.00 & 41 & 0.08 & 0.92 & 0.00 \\
\hline 19 & 0.15 & 0.85 & 0.00 & 42 & 0.32 & 0.68 & 0 \\
\hline 20 & 0.06 & 0.94 & 0.00 & 43 & 0.25 & 0.75 & 0 \\
\hline 21 & 0.00 & 1.00 & 0.00 & 44 & 0.75 & 0.25 & 0 \\
\hline 22 & 0.05 & 0.95 & 0.00 & 45 & 1 & 0 & 0 \\
\hline 23 & 0.10 & 0.90 & 0.00 & & & & \\
\hline
\end{tabular}

Table S5. Automated shape classification for PDMAEMA- $b$-PHPMA nanostructures. 


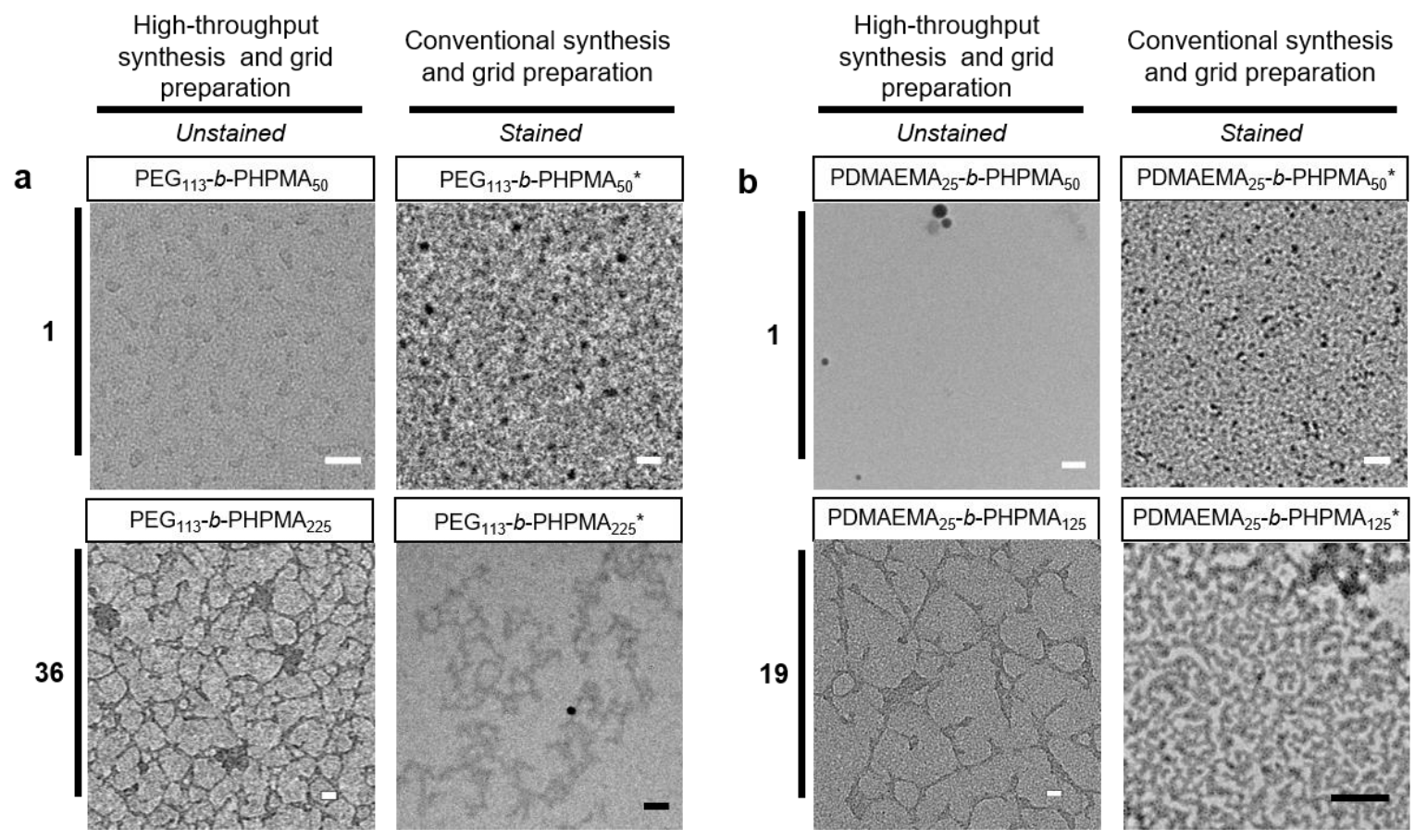

Figure S4. Companion to Figure 7 of the main text. Here, comparing spherical micelles prepared by 96-well plate, with conventional scale-up, and comparison of worm shaped micelles in the same fashion. a) TEM images of Composition 1 and 36 PEG- $b$-PHPMA nanostructures generated in a 96-well plate, dispensed with the sciTEM (left) or prepared by hand from a scaled-up (250 $\mathrm{mg}$ ) sample with uranyl acetate stain (right). b) TEM images of Composition 1 and 19 PDMAEMA-b-PHPMA nanostructures generated in a 96-well plate, dispensed using the sciTEM (left) or prepared by hand from a scaled-up (250 $\mathrm{mg}$ ) sample with uranyl acetate stain (right). Starred compositions denote the scaled-up sample. White scale bars $=20 \mathrm{~nm}$, black scale bars $=500 \mathrm{~nm}$. 


\section{Supporting References}

(1) Touve, M. A.; Figg, C. A.; Wright, D. B.; Park, C.; Cantlon, J.; Sumerlin, B. S.; Gianneschi, N. C. Polymerization-Induced Self-Assembly of Micelles Observed by Liquid Cell Transmission Electron Microscopy. ACS Cent. Sci. 2018, 4, 543-547.

(2) Mastronarde, D. N. Automated Electron Microscope Tomography Using Robust Prediction of Specimen Movements. J. Struct. Biol. 2005, 152, 36-51.

(3) Vo, G. D.; Park, C. Robust Regression for Image Binarization under Heavy Noise and Nonuniform Background. Pattern Recognit. 2018, 81, 224-239.

(4) Srivastava, A.; Klassen, E.; Joshi, S. H.; Jermyn, I. H. Shape Analysis of Elastic Curves in Euclidean Spaces. IEEE Trans. Pattern Anal. Mach. Intell. 2011, 33 (7), 1415-1428. 